\title{
Cyclopamine tartrate, an inhibitor of Hedgehog signaling, strongly interferes with mitochondrial function and suppresses aerobic respiration in lung cancer cells
}

Md Maksudul Alam', Sagar Sohoni ${ }^{1}$, Sarada Preeta Kalainayakan ${ }^{1}$, Massoud Garrossian ${ }^{2}$ and Li Zhang ${ }^{1,3^{*}}$

\begin{abstract}
Background: Aberrant Hedgehog $(\mathrm{Hh})$ signaling is associated with the development of many cancers including prostate cancer, gastrointestinal cancer, lung cancer, pancreatic cancer, ovarian cancer, and basal cell carcinoma. The Hh signaling pathway has been one of the most intensely investigated targets for cancer therapy, and a number of compounds inhibiting Hh signaling are being tested clinically for treating many cancers. Lung cancer causes more deaths than the next three most common cancers (colon, breast, and prostate) combined. Cyclopamine was the first compound found to inhibit Hh signaling and has been invaluable for understanding the function of $\mathrm{Hh}$ signaling in development and cancer. To find novel strategies for combating lung cancer, we decided to characterize the effect of cyclopamine tartrate (CycT), an improved analogue of cyclopamine, on lung cancer cells and its mechanism of action.
\end{abstract}

Methods: The effect of CycT on oxygen consumption and proliferation of non-small-cell lung cancer (NSCLC) cell lines was quantified by using an Oxygraph system and live cell counting, respectively. Apoptosis was detected by using Annexin V and Propidium lodide staining. CycT's impact on ROS generation, mitochondrial membrane potential, and mitochondrial morphology in NSCLC cells was monitored by using fluorometry and fluorescent microscopy. Western blotting and fluorescent microscopy were used to detect the levels and localization of Hh signaling targets, mitochondrial fission protein Drp1, and heme-related proteins in various NSCLC cells.

Results: Our findings identified a novel function of CycT, as well as another Hh inhibitor SANT1, to disrupt mitochondrial function and aerobic respiration. Our results showed that CycT, like glutamine depletion, caused a substantial decrease in oxygen consumption in a number of NSCLC cell lines, suppressed NSCLC cell proliferation, and induced apoptosis. Further, we found that CycT increased ROS generation, mitochondrial membrane hyperpolarization, and mitochondrial fragmentation, thereby disrupting mitochondrial function in NSCLC cells. (Continued on next page)

\footnotetext{
*Correspondence: li.zhang@utdallas.edu

'Department of Molecular and Cell Biology, Center for Systems Biology, University of Texas at Dallas, Mail Stop RL11, 800 W. Campbell Road, Richardson, TX 75080, USA

${ }^{3}$ The Cecil H. and Ida Green Distinguished Chair, Department of Biological Sciences, The University of Texas at Dallas, Mail Stop RL11, 800 W. Campbell Road, Richardson, TX 75080, USA

Full list of author information is available at the end of the article
} International License (http://creativecommons.org/licenses/by/4.0/), which permits unrestricted use, distribution, and reproduction in any medium, provided you give appropriate credit to the original author(s) and the source, provide a link to the Creative Commons license, and indicate if changes were made. The Creative Commons Public Domain Dedication waiver (http://creativecommons.org/publicdomain/zero/1.0/) applies to the data made available in this article, unless otherwise stated. 
(Continued from previous page)

Conclusions: Together, our work demonstrates that CyCT, and likely other Hh signaling inhibitors, can interrupt NSCLC cell function by promoting mitochondrial fission and fragmentation, mitochondrial membrane hyperpolarization, and ROS generation, thereby diminishing mitochondrial respiration, suppressing cell proliferation, and causing apoptosis. Our work provides novel mechanistic insights into the action of Hh inhibitors in cancer cells.

Keywords: Non-small-cell lung cancer (NSCLC), Hedgehog signaling, Cyclopamine tartrate, SANT1, Oxygen consumption, Mitochondrial fragmentation, ROS, Apoptosis, Glutamine depletion

\section{Background}

Hedgehog (Hh) signaling is a key regulator of development and stem cell fate in animals [1]. Dysregulation of the Hh pathway is responsible for various developmental malformations, such as holoprosencephaly [2]. Aberrant activation of the Hh signaling is implicated in a variety of cancers, such as prostate cancer, gastrointestinal cancer, lung cancer, pancreatic cancer, ovarian cancer, and basal cell carcinoma [3-8]. Therefore, Hh signaling pathway has become a therapeutic target for treating many types of cancers. Particularly, a great deal of efforts have been focused on targeting smoothened (SMO), a G protein-coupled receptor mediating Hedgehog $(\mathrm{Hh})$ signaling [9]. Many SMO inhibitors have been generated and tested, and all have shown efficacy as anti-tumor agents [10]. For example, vismodegib is the first FDAapproved SMO inhibitor for the treatment of advanced and metastatic basal cell carcinoma. Currently, vismodegib and many other SMO inhibitors are being investigated in clinical trials in a range of advanced cancers $[9,10]$. Consequently, understanding the molecular actions of such inhibitors can be of great value to the improvement of therapeutic strategies for many types of cancers.

The first identified inhibitor of Hh signaling was cyclopamine, a molecule isolated from corn lilies [11, 12]. Cyclopamine binds to and inhibits SMO. Cyclopamine has been very valuable for understanding the function of Hh signaling and has been widely used as an Hh inhibitor in cell and murine models of various tumors [3-5, 13-15]. However, the usefulness of cyclopamine (Суc) as a therapeutic drug is hindered by its poor aqueous solubility [16]. To improve the solubility and efficacy of Cyc, Dr. Garrossian, a contributor of this manuscript, generated cyclopamine tartrate (СуcT) [17]. Indeed, CycT is water soluble, and its activity in inhibiting Hh signaling is higher than Cyc. Furthermore, CycT is effective in causing tumor shrinkage in two mouse models of basal cell carcinomas [17]. Therefore, we decided to examine the efficacy of CycT in inhibiting the proliferation and function of an array of non-small-cell lung cancer (NSCLC) cell lines.

Previously, using a matched pair of cell lines representing normal nonmalignant and NSCLC cells developed from the same patient, we found that oxygen consumption is intensified in NSCLC cells and tumors [18]. Specifically, the rates of both glucose and oxygen consumption in NSCLC HCC4017 cells are elevated, with the elevation of oxygen consumption greater than that of glucose consumption. Inhibition of mitochondrial respiration interferes strongly with NSCLC cell function, proliferation and migration [18]. In this paper, we sought ways to suppress aerobic respiration, which may lead to novel therapeutic strategies to treat lung cancer and other cancers as well. Firstly, we found that oxygen consumption is intensified in an array of NSCLC cell lines. Secondly, we demonstrated that CycT and another SMO inhibitor SANT1, like glutamine depletion, suppress the rates of oxygen consumption and the rates of cancer cell proliferation. Further analyses of various cellular functions showed that CycT, as well as SANT1, increases ROS generation and mitochondrial membrane potential in NSCLC cells. Ultimately, these SMO inhibitors cause mitochondrial fragmentation, leading to apoptosis. Our studies uncovered a novel mode of anticancer action of an Hh inhibitor, which may have broad implications in the development and application of many Hh inhibitors currently being tested.

\section{Results}

Cyclopamine tartrate (СусT), like glutamine depletion, strongly suppresses oxygen consumption in NSCLC cells

Previously, we showed that the rate of oxygen consumption in NSCLC HCC4017 cells is intensified compared to the nonmalignant HBEC cells representing normal lung epithelial cells from the same patient [18], as shown in Fig. 1a. Additionally, we measured oxygen consumption rates in five other NSCLC cell lines and found that they were substantially increased in all NSCLC cell lines (see Fig. 1a). Furthermore, we examined the effect of glucose depletion, glutamine depletion, and Сyc T on the rates of oxygen consumption in these NSCLC cell lines. We found that glucose depletion generally enhances oxygen consumption rates, while glutamine depletion diminishes the rates (see Fig. 1b-g). Glucose and glutamine are two critical fuels for cancer cells [19, 20]. When glucose is limiting, the cells use glutamine, and vice versa. Thus, these results showed that in the absence of glucose, glutamine supports intensified oxygen consumption in cancer cells. In the absence of glutamine, even in the 

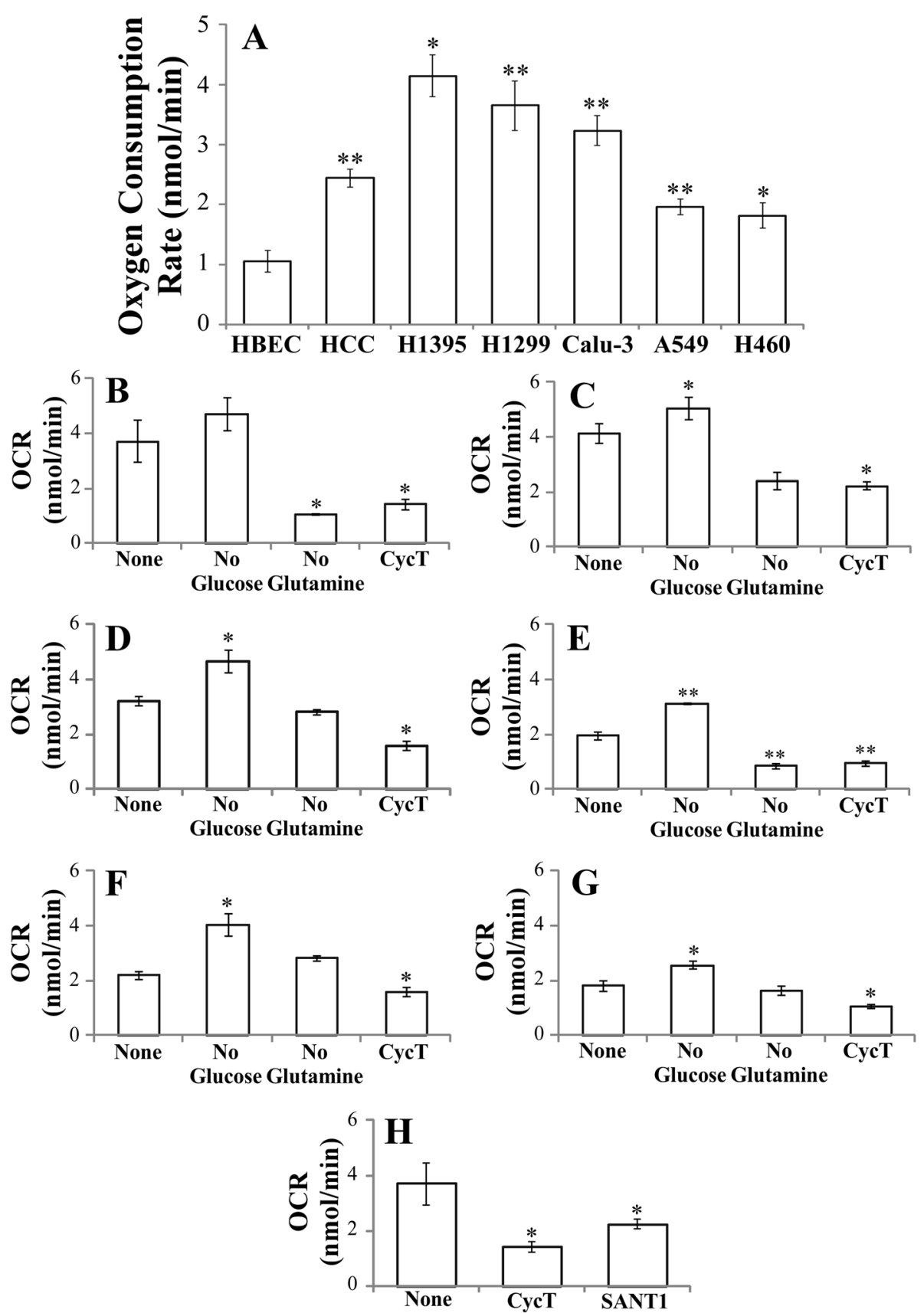

Fig. 1 a The rates of oxygen consumption are intensified in various NSCLC cell lines. b-g CycT, like glutamine depletion, strongly diminished oxygen consumption rates in NSCLC H1299 (b), H1395 (c), Calu-3 (d), A549 (e), HCC4017 (f), and H460 (g) cells. NSCLC cell lines were cultured in their normal medium or in medium lacking glucose or glutamine, or treated with CycT, as indicated. h SMO inhibitor SANT1, like CycT, can diminish oxygen consumption in NSCLC cells. H1299 cells were treated with CycT or SANT1. The rates of oxygen consumption were measured. The data shown were averages of at least three independent measurements. For statistical analysis, the values were compared to that in nontumorigenic HBEC lung cells (a) or those in normal culture medium (in B-G), by using Welch 2-sample $t$-test. *, $p$ value $<0.05$; **, $p$ value $<0.005$

presence of glucose, oxygen consumption was substantially reduced (Fig. 1b-g). Notably, CycT diminished the rates of oxygen consumption in cancer cells largely to the same extent as glutamine depletion. Likewise, another SMO inhibitor SANT1 [21, 22] diminished oxygen consumption in NSCLC cells, as expected. These results indicate that CycT can cause the same effect as glutamine depletion on cancer cell metabolism and aerobic respiration.

\section{CycT causes apoptosis in NSCLC cells}

The data shown above revealed a strong effect of CycT on aerobic respiration. Thus, we further examined the 
effect of CycT on NSCLC cell proliferation. We found that CycT diminishes the proliferation and survival of NSCLC cells, although the sensitivity of different cell lines to CycT varies (see Additional file 1: Fig. S1). We also tested whether CycT causes apoptosis in NSCLC cells by using Annexin $\mathrm{V}$ and propidium iodide (PI) staining. We found that CycT indeed causes apoptosis in NSCLC cells, albeit with varying efficacy in different NSCLC cell lines. For example, after $24 \mathrm{~h}$ of treatment with CycT, H1299 cells were mostly apoptotic, as detected by Annexin V staining (Fig. 2a). PI staining further showed that a fraction of these apoptotic H1299 cells were in the late apoptotic stage. A549 cells, as shown by the proliferation rates in Additional file 1: Fig. S1, were more resistant to CycT (see Fig. 2b). After $24 \mathrm{~h}$ of treatment, only a fraction of the cells showed signs of apoptosis, as detected by Annexin V staining. No A549 cells were in late apoptotic stage (see Fig. 2b). Nonetheless, our results showed that CycT can cause apoptosis in NSCLC cells. Notably, another SMO inhibitor

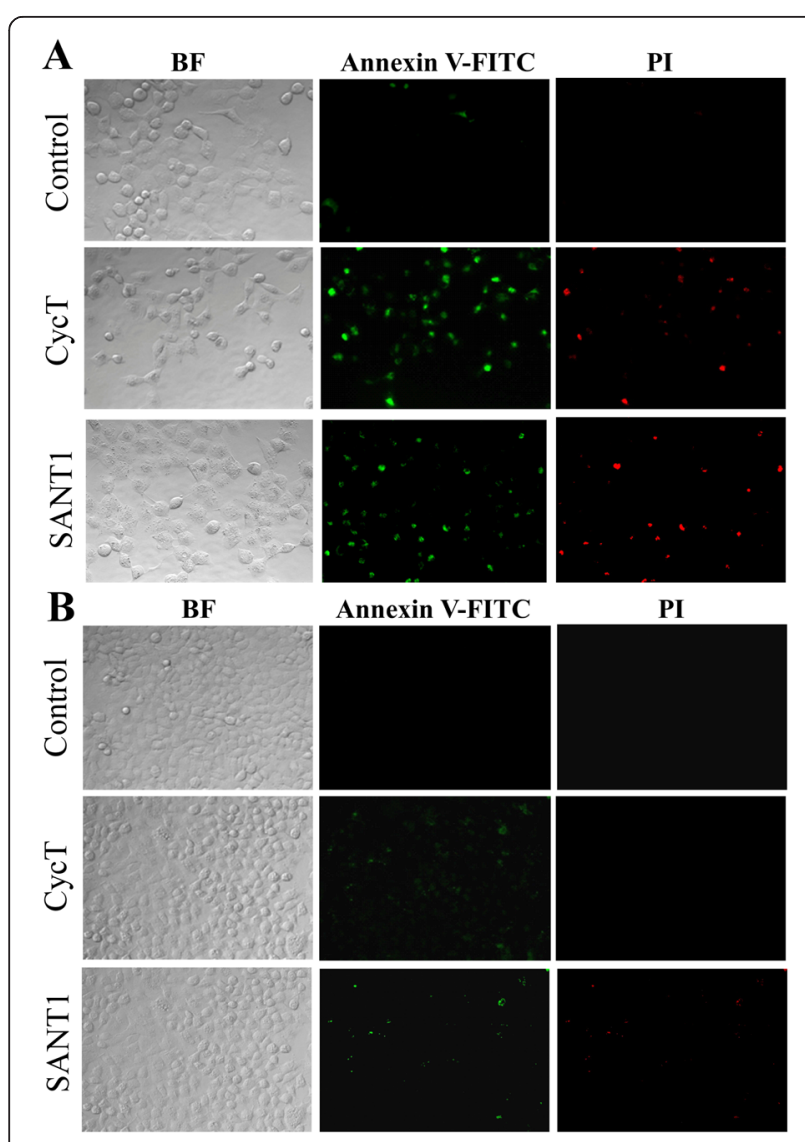

Fig. 2 CycT and SANT1 induce apoptosis in H1299 (a) and A549 (b) NSCLC cell lines. The NSCLC cells were treated with CycT or SANT1 for $24 \mathrm{~h}$. Then cells were subjected to apoptosis assay by using Annexin V-FITC and Propidium lodide (PI) staining. The images of cells were captured with bright field microscopy (BF) or with fluorescent microscopy with a FITC or rhodamine (for PI) filter
SANT1, like CycT, also exerted similar effects on NSCLC cells (Fig. 2a and b).

\section{CycT does not exert a considerable effect on heme metabolism}

Heme is a central factor in aerobic respiration and oxidative phosphorylation [23]. Previously, we have shown that limiting intracellular heme levels strongly diminishes mitochondrial respiration and NSCLC cell proliferation and migration [18]. Therefore, we examined whether CycT impacts heme synthesis and metabolism. We found that CycT does not significantly affect the rate of heme synthesis in NSCLC cells (data not shown). Likewise, we found that CycT does not significantly affect the protein levels of the rate-limiting heme synthetic enzyme ALAS1 and the degradation enzyme HO1 (see Fig. 3a and b). For a control, we showed that CycT reduces the level of the Hh signaling target Gli1 (Fig. 3c), as expected. Furthermore, we found that CycT treatment reduced the levels of phosphorylated p44/42 MAPK. The activation of p44/42 MAPK signaling pathway has been shown to be critical for Hh signaling previously [24]. These results show that CycT does not affect aerobic respiration by impacting heme metabolism.

\section{CycT increases ROS generation and interferes with mitochondrial function in NSCLC cells}

To further investigate the mode by which CycT causes NSCLC cell death, we measured ROS generation in CycT-treated and untreated NSCLC cells. We found that CycT causes a substantial increase in ROS generation in NSCLC cells, including H1299 (Fig. 4a), A549 (Fig. 4b), and $\mathrm{H} 460$ (Fig. 4c) cells. In addition, we found that another SMO inhibitor SANT1 increased ROS generation in NSCLC cells (Fig. 4d). Because of the dominant role of mitochondria in oxygen metabolism, mitochondria are mainly responsible for the generation of cellular ROS. Therefore, we also examined the effect of CycT on mitochondrial function. First, we measured and compared mitochondrial membrane potential in CycT-treated and CycT-untreated cells. We found that CycT increases mitochondrial membrane potential substantially in NSCLC cells (Fig. 5a, b and c). This effect of CycT appeared to be stronger in H1299 (Fig. 5a) and H460 (Fig. 5c) cells than in A549 cells (Fig. 5b). Likewise, another SMO inhibitor SANT1 increased mitochondrial membrane potential in NSCLC cells (Fig. 5d).

\section{CycT causes mitochondrial fragmentation in NSCLC cells}

Mitochondrial morphology is closely linked to mitochondrial function [25]. Changes in mitochondrial morphology regulate many mitochondrial functions, such as the respiratory activity of the electron transport chain and apoptosis [26]. We therefore examined the effect of CycT on 
A
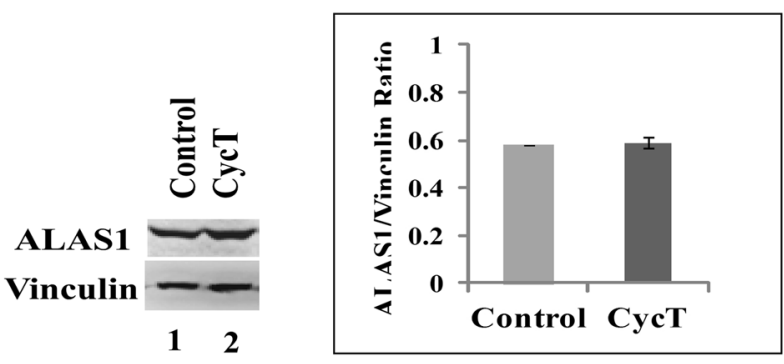

B

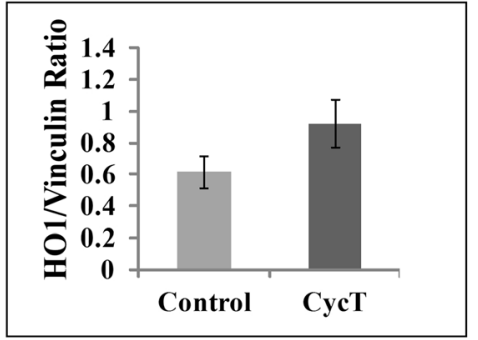

C
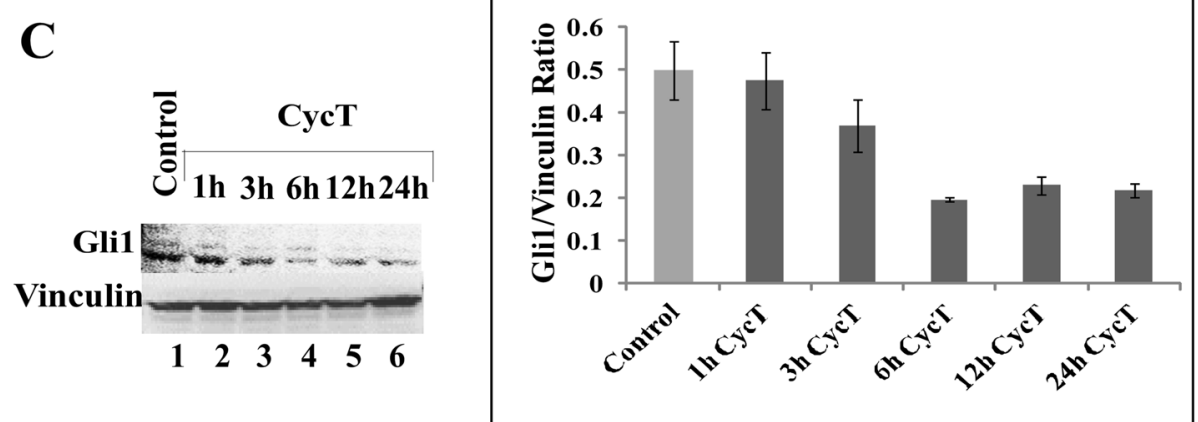

D
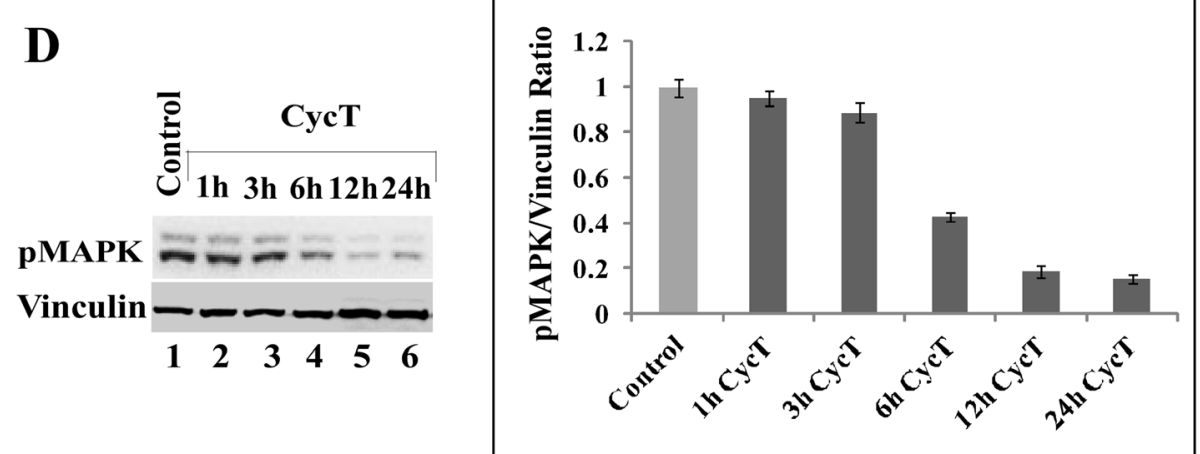

Fig. 3 The effect of CycT treatment on the levels of ALAS1 (a), HO1 (b), Gli1 (c), and phospho-p44/p42 MAPK (d). The NSCLC A549 cells were cultured and treated with CycT for $24 \mathrm{~h}$ [lane 2 in (a) and (b)] or without CycT [lane 1 in (a) and (b)]. In (c) and (d), cells were treated without (lane 1) or with CycT for 1 (lane 2), 3 (lane 3), 6 (lane 4), 12 (lane 5), and 24 (lane 6) hours as indicated. Protein extracts were prepared and the levels of the proteins were detected by Western blotting. The protein level of vinculin was used for normalization. For statistical analysis, the levels in treated cells were compared to the levels in untreated cells, by using Welch 2 -sample $t$-test. ${ }^{*}, p$ value $<0.05$

mitochondrial morphology in NSCLC cells by using MitoTracker Red. Fig. 6 shows that CycT treatment caused mitochondrial fragmentation in NSCLC cells, H1299 (Fig. 6a), A549 (Fig. 6b), and H460 (Fig. 6c) cells. SANT1 exerted similar effects on the NSCLC cells (not shown). Because Drp1 is the main protein promoting mitochondrial fission and fragmentation [27], we also examined the effect of CycT on Drp1 distribution. Additional file 2: Fig. S2 shows that in CycT-treated NSCLC H1299 (Additional file 2: Fig. S2A) and A549 (Additional file 2: Fig. S2B) cells, Drp1 were indeed selectively localized to various mitochondrial fission sites: The localization pattern of Drp1 

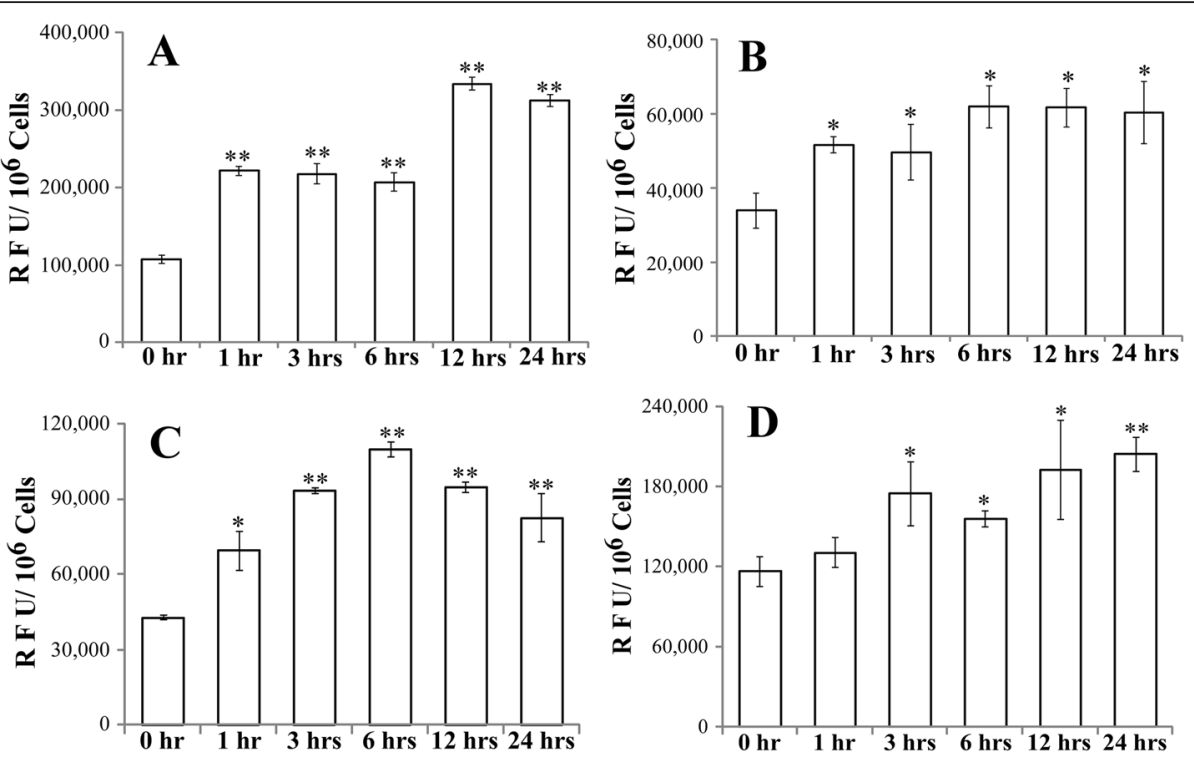

Fig. 4 CycT treatment increases ROS production in NSCLC H1299 (a), A549 (b), and H460 (c) cells. d SANT1 treatment also increases ROS production in NSCLC H1299 cells. NSCLC cells were treated with CycT or SANT1 for the indicated time periods. Then cells were incubated with 2,7-dichlorodihydrofluorescein diacetate (DCFH-DA) for $30 \mathrm{~min}$. Fluorescence intensity was measured and normalized according to cell density. For statistical analysis, the levels in CycT-treated cells were compared to the levels in untreated cells, by using Welch 2-sample t-test. *, $p$ value $<0.05$; **, $p$ value $<0.005$
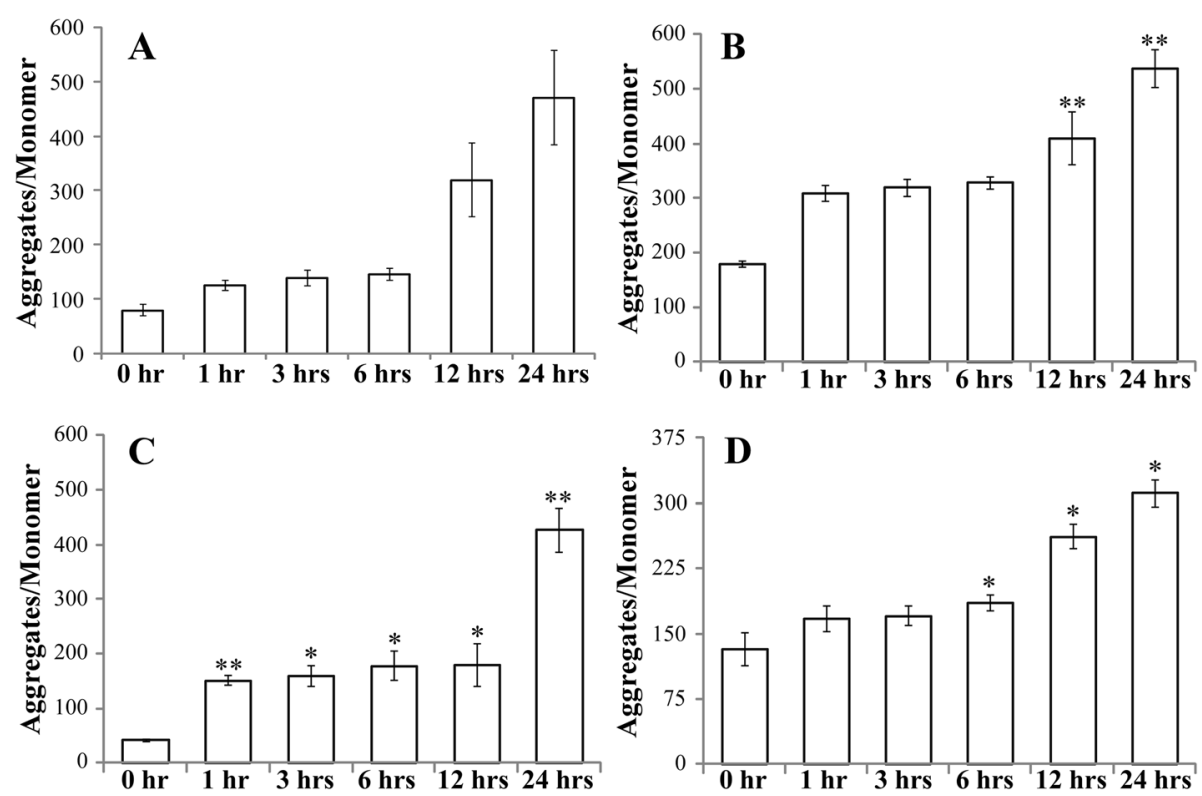

Fig. 5 The effect of CycT treatment on mitochondrial membrane potential in NSCLC H1299 (a), A549 (b), and H460 (c) cells. d SANT1 treatment also increases mitochondrial membrane potential in NSCLC H1299 cells. NSCLC cells were treated with CycT or SANT1 for the indicated time periods. Then mitochondrial membrane potential in these cells was measured by using JC-1 staining. Mitochondrial membrane potential was expressed as the ratio of aggregates/monomer, which was calculated by dividing red fluorescence intensity with green fluorescence intensity. For statistical analysis, the levels in CycT-treated cells were compared to the levels in untreated cells, by using Welch 2-sample $t$-test. *, $p$ value $<0.05$; ${ }^{*}, p$ value $<0.005$ 


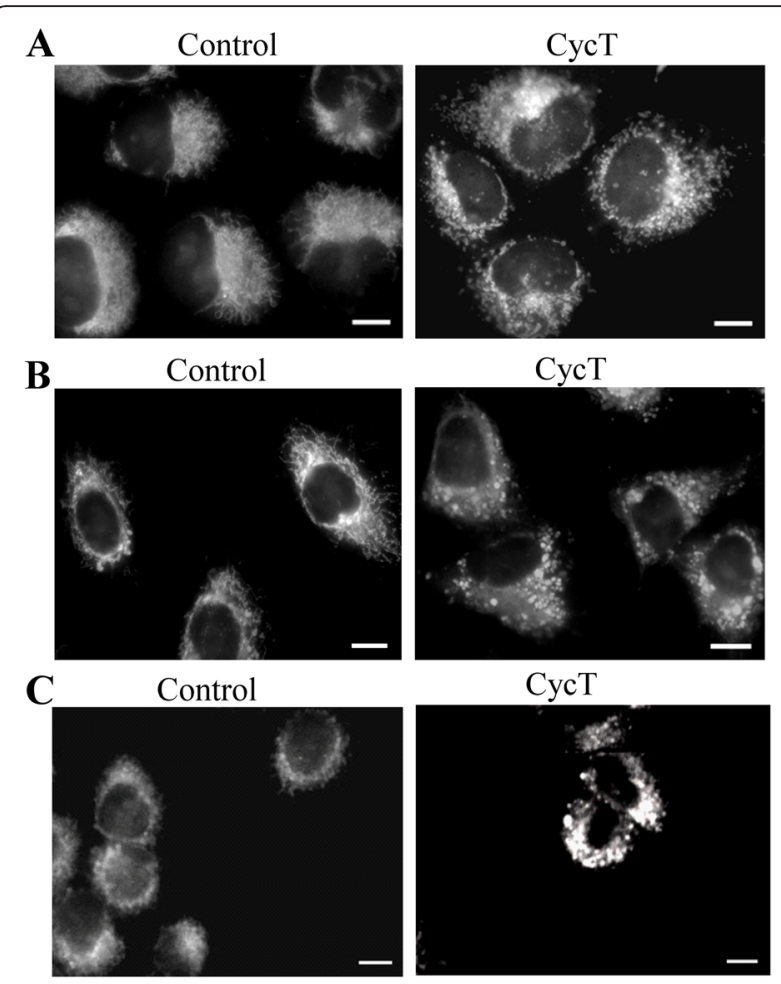

Fig. 6 CycT treatment causes mitochondrial fragmentation in NSCLC H1299 (a), A549 (b), and H460 (c) cells. NSCLC cells were treated with CycT for $24 \mathrm{~h}$, and then stained with MitoTracker Red. Fluorescent images were acquired and shown here. The scale bar indicates $10 \mu \mathrm{m}$

completely coincided with the MitoTracker Red staining pattern. These results demonstrated that CycT can induce Drp1 to promote mitochondrial fission and fragmentation.

\section{Discussion}

Targeting Hh signaling is an important strategy that is being developed to treat a variety of metastatic and advanced cancers $[9,28]$. Vismodegib, a SMO antagonist, was the first $\mathrm{Hh}$ inhibitor to receive approval from the USA FDA in January 2012 for the treatment of locally advanced or metastatic basal cell carcinoma (BCC) [29]. Currently, NIH lists 91 ongoing or completed clinical trials testing $\mathrm{Hh}$ inhibitors against a variety of cancers (https://clinicaltrials.gov/ct2/results?term=HEDGEHOG). Therefore, a comprehensive understanding of the modes of Hh inhibitor action can benefit the treatment of a wide range of cancers. Here, by using the well-studied $\mathrm{Hh}$ inhibitor cyclopamine tartrate, we identified a new activity of this drug against aerobic respiration and mitochondrial function in NSCLC cancer cells.

First, we found that oxygen consumption is intensified in an array of NSCLC cell lines and that glutamine has a prominent role in promoting oxygen consumption, because glucose depletion in the presence of glutamine enhanced oxygen consumption while glutamine depletion in the presence of glucose diminished oxygen consumption substantially (Fig. 1). This is entirely consistent with previous studies showing glutamine as the dominant respiratory substrate in tumor cells [30,31]. Remarkably, we found that CycT exerted largely the same effect on oxygen consumption as glutamine depletion (see Fig. 1b-g). Glutamine provides a crucial source of cellular energy and building blocks for cancer cells, and targeting glutamine uptake and metabolism has become an important approach in treating drug-resistant cancers [32, 33]. Our finding linking both CycT treatment and glutamine depletion with diminished aerobic respiration provides a new way to control cancer cell bioenergetics. Although different NSCLC cell lines exhibited varying rates of oxygen consumption (Figs. 1b-g), CycT, like glutamine depletion, strongly diminished oxygen consumption in all cell lines, suggesting that CycT can be an effective inhibitor of cellular energy production in all these lines. The same effect of glutamine depletion and CycT treatment suggests that $\mathrm{Hh}$ inhibitors may be used in substitution of drugs targeting glutamine metabolism in cancer therapy.

Second, we showed that CycT strongly suppressed NSCLC cell proliferation (Additional file 1: Fig. S1) and induced apoptosis (Fig. 2a and b). Notably, apoptosis of NSCLC cells was preceded by increased ROS generation (Fig. 4a and b) and increased mitochondrial membrane potential (Fig. 5a and b). At first sight, increased mitochondrial membrane potential accompanying apoptosis may seem paradoxical. However, hyperpolarization of mitochondrial membrane potential induced by various stress factors have been observed in a wide array of cells ranging from neuronal to blood cells [34]. For example, both glucose and oxygen deprivation induces mitochondrial membrane potential hyperpolarization [35]. Hyperpolarization of mitochondrial membrane potential will lead to excessive ROS production [34, 36]. The connection between the $\Delta \Psi \mathrm{m}$ and ROS is exponential when $\Delta \Psi \mathrm{m}$ exceeds $140 \mathrm{mV}$ [37]. Ultimately, increased ROS generation will lead to apoptosis [38, 39], as indicated in Figs. 4 and 5.

Third, we found that CycT strongly induced mitochondrial fission and fragmentation (Fig. 6). As expected, CycT-induced mitochondrial fragmentation accompanied the recruitment of Drp1 to the mitochondrial outer membrane and fission sites (see Additional file 2: Fig. S2), consistent with previous models of mitochondrial fission [27]. Mitochondrial fragmentation is often associated with apoptosis, although it can occur in a variety of conditions independently of apoptosis [25, 40]. Here our results suggest that CycT-induced apoptosis in NSCLC cells is associated with mitochondrial fragmentation. Interestingly, while CycT caused strong mitochondrial fragmentation in both A549 and H1299 cells, apoptosis was much less prominent in A549 cells (Fig. 2), 
suggesting differential sensitivity of NSCLC cells to mitochondrial fragmentation and CycT.

In sum, our results provide novel insights into the potential mechanisms of action of Hh signaling pathway inhibitors. Previous studies have shown that Gli transcriptional factors are the key mediators of Hh signaling $[1,2]$. Furthermore, genome-wide analyses using gene expression profiling and chromatin immunoprecipitation have identified many Gli target genes [41-43]. A careful examination of these target genes showed that there are 30 genes encoding for mitochondrial functions, such as MRPL23, GLUL, SLC25A13, PRDX6, and ATP6V1E1 [41]. These targets also include NDUFS8, a subunit of mitochondrial NADH:ubiquinone oxidoreductase, or Complex I; Cyb5b, a cytochrome b5 outer mitochondrial membrane isoform; 1810063B05Rik, cytochrome c oxidase assembly factor 6; and catalase. Although the identification of such a relatively small number of mitochondrial targets does not allow the inference of a global effect of Hh signaling or Hh inhibitors on mitochondria, it does lend support to our new finding that the Hh inhibitor CycT impacts mitochondrial morphology and function, thereby modulating mitochondrial respiration and apoptosis. Further studies comparing SMO inhibitors, such as CycT, and Gli inhibitors may provide additional insights into which proteins in the Hh signaling pathway play a dominant role in mitochondrial morphology and function.

\section{Conclusion}

The major findings of our study are: a) CycT, like glutamine depletion, causes a substantial decrease in oxygen consumption in a number of NSCLC cell lines; b) CycT suppresses proliferation and induces apoptosis in NSCLC cells; c) CycT and evidently other Hh inhibitors promote mitochondrial fission and fragmentation, mitochondrial membrane hyperpolarization, and ROS generation; and d) Hh signaling inhibitors can act on mitochondria and cause broad and dramatic changes in mitochondrial morphology, respiration and function. These new findings can shed light on the mechanisms underlying various cancers associated with aberrant Hh signaling and can provide novel insights into how to optimize anti-Hh signaling strategy for treating cancer.

\section{Methods}

\section{Lung cell lines, antibodies and reagents}

HBEC30KT and HCC4017 cell lines representing normal and NSCLC cells $[44,45]$ were provided by Dr. John Minna's lab (UTSW) as a gift. They were developed from the same patient and were maintained in ACL4 supplemented with $2 \% \mathrm{FBS}$ under $5 \% \mathrm{CO}_{2}$ at $37{ }^{\circ} \mathrm{C}$ [45]. All other NSCLC cell lines, H1395, H1299, Calu-3, A549 and $\mathrm{H} 460$ were purchased from ATCC, and were maintained according to ATCC procedures. All tissue culture media, including those lacking glucose or glutamine, were purchased from Invitrogen Life Technologies. HBEC30KT, HCC4017, H1299 and A549, were authenticated by using the services provided by Genetica DNA Laboratories, and they were found to be $100 \%$ match. Other NSCLC cell lines were used for measurements immediately following the purchase. Cyclopamine tartrate (СуcT) was provided by Logan Natural Products (Plano, Texas). SANT1 was purchased from Santa Cruz Biotechnology. For measuring the effect of CycT or SANT1 on lung cell proliferation, cells were seeded in 48 -well plate at a density of $10^{4}$ cells/well. After culturing for $24 \mathrm{~h}$, cells were treated with the indicated concentrations for $24 \mathrm{~h}$ or the indicated time points. At the indicated times, the number of live cells was counted by using trypan blue staining and a hemocytometer. Polyclonal anti-ALAS1, anti-HO1, anti-Gli1 and anti-Drp1 were purchased from Santa Cruz Biotechnology. Monoclonal anti-vinculin antibody was purchased from Sigma-Aldrich. Polyclonal anti-phospho-p44/42 MAPK antibody was purchased from Cell Signaling Technology.

\section{Measurement of oxygen consumption rates}

NSCLC cells ( 70 \% confluence) were maintained in medium with $25 \mu \mathrm{M}$ CycT or $50 \mu \mathrm{M}$ SANT1, or in medium lacking glucose or glutamine for $24 \mathrm{~h}$. Then oxygen consumption was measured, as described previously [46]. Briefly, cells with about $80 \%$ confluence were trypsinized and resuspended in fresh, air-saturated medium. For each measurement, $10^{6}$ cells (in $350 \mu \mathrm{l}$ ) were introduced in the chamber of an Oxygraph system (Hansatech Instruments), with a Clark-type electrode placed at the bottom of the respiratory chamber. During measurements, the chamber was thermostated at $37^{\circ} \mathrm{C}$ by a circulating water bath. An electromagnetic stirrer bar was used to mix the contents of the chamber. Each measurement was replicated at least three times. Standard deviations were calculated, and $\mathrm{p}$ values were calculated by using Welch 2-sample $t$-test (R program).

\section{Measurement of ROS generation and mitochondrial membrane potential}

The intracellular ROS levels were measured by using 2,7-dichlorodihydrofluorescein diacetate (DCFH-DA) (Cayman Chemical), which is oxidized into highly fluorescent 2,7- dichlorofluorescein in the presence of intracellular ROS. Cells were seeded in 96-well plates with black walls and clear bottoms at a density of 10,000 cells per well. Cells were treated with $25 \mu \mathrm{M}$ CycT or $50 \mu \mathrm{M}$ SANT1 for the indicated times. Cells were then incubated for 30 min with $10 \mu \mathrm{M}$ DCFH-DA dissolved in fresh media. Cells were then rinsed twice with PBS, and each well was filled with $100 \mu \mathrm{l}$ PBS. Fluorescence was detected by using a fluorescent plate reader (Bio Tek, 
Synergy Mx microplate reader) with the excitation and emission wavelengths at 490 and $535 \mathrm{~nm}$, respectively.

Changes in the mitochondrial membrane potential $(\Delta \Psi \mathrm{m})$ were measured quantitatively by staining with the cationic dye JC-1(Molecular probes), which accumulates in the mitochondria, showing green fluorescence at lower membrane potential and forming J-aggregates with red fluorescence at higher membrane potential. Cells were seeded in 96 well black wall and clear bottom plates at a density of 10,000 cells per well. Cells were treated with $25 \mu \mathrm{M}$ CycT or $50 \mu \mathrm{M}$ SANT1 for the indicated times. Cells were then incubated with $200 \mu \mathrm{l}$ of fresh medium containing $2 \mu \mathrm{g} / \mu \mathrm{l}$ of JC-1 dye for $30 \mathrm{~min}$ in the dark. The cells were washed twice with PBS, and the plates were immediately read with a fluorescent plate reader (Bio Tek, Synergy Mx microplate reader) with excitation and emission wavelengths set at 540 and $595 \mathrm{~nm}$, respectively, for red fluorescence; and 485 and $535 \mathrm{~nm}$, respectively, for green fluorescence.

\section{Preparation of protein extracts and Western blotting}

NSCLC cells were treated, collected, and lysed by using the RIPA buffer (Cell Signaling Technology) containing the protease inhibitor cocktail. Protein concentrations were determined by using the BCA assay kit (Thermo Scientific). $50 \mu \mathrm{g}$ of proteins from each treatment condition were electrophoresed on $9 \%$ SDS-Polyacrylamide gels, and then transferred onto the Immuno-Blot PVDF Membrane (Bio-Rad). The membranes were probed with polyclonal antibodies, followed by detection with a chemiluminescence Western blotting kit (Roche Diagnostics). The signals were detected by using a Carestream image station 4000MM Pro, and quantitation was performed by using the Carestream molecular imaging software version 5.0.5.30 (Carestream Health, Inc.). Antibodies used were purchased from Santa Cruz Biotechnology and Sigma-Aldrich.

\section{Mitochondria imaging and indirect immunofluorescence staining}

Mitochondria were visualized by using Mito Tracker Red CMXRos (Molecular probes), which passively diffuses across the plasma membrane and accumulates in active mitochondria. Cells were grown on chamber slides and treated with CycT for $24 \mathrm{~h}$. Cells were then stained with $200 \mathrm{nM}$ of CMXRos in complete growth medium for $30 \mathrm{~min}$ at $37^{\circ} \mathrm{C}$, washed with prewarmed PBS three times, and fixed with $4 \%$ formaldehyde in PBS for $10 \mathrm{~min}$. After washing twice with PBS the slides were covered, and fluorescent images were captured with a multi-channel Zeiss Axio Observer Z1 fluorescent microscope with a Zeiss 40X Oil immersion lens and with a high speed AxioCam MRm Rev3 monochrome camera.
Indirect immunofluorescence staining with Drp1 antibodies (purchased from Santa Cruz Biotechnology) was performed by following the procedures provided by the antibody manufacturer. FITC and DAPI fluorescent images were captured by using a multi-channel Zeiss Axio Observer Z1 fluorescent microscope. Apoptosis was detected by using the ApoAlert Annexin V-FITC Apoptosis Kit (Clontech). Cells were seeded in a 96-well black wall clear bottom plate at the density of 10,000 cells per well. After one day, cells were treated with $25 \mu \mathrm{M}$ CycT or SANT1 in fresh medium. Twenty four hours post treatment, apoptosis assay was performed according to manufacturer's protocol. Fluorescent images were captured using a multi-channel Zeiss Axio Observer Z1 fluorescent microscope with a Zeiss 20X lens and with a high speed AxioCam MRm Rev3 monochrome camera.

\section{Ethics approval and consent to participate}

There was not any ethics approval or consent required for the use of human derived cell lines in this study.

\section{Additional files}

\begin{abstract}
Additional file 1: Figure S1. The effect of CycT treatment on NSCLC cancer cell proliferation. \%live cells was calculated by dividing the number of treated cells with the number of untreated cells (seeded with the same number of cells). It shows the relative proliferative rates of treated cells (10 or $25 \mu \mathrm{M})$ vs. untreated cells (None). For statistical analysis, the values for treated cells were compared to the values for untreated cells, by using Welch 2 -sample $t$-test. ${ }^{*}, p$ value $<0.05 ;{ }^{* *}, p$ value $<0.005$. (JPG $270 \mathrm{~kb}$ )
\end{abstract}

Additional file 2: Figure S2. Drp1 localizes to the mitochondrial fission sites in CycT-treated NSCLC H1299 (A) and A549 (B) cells. NSCLC cells were treated with CycT for $24 \mathrm{~h}$. Cells were incubated with anti-Drp1 antibodies, and then with FITC-conjugated goat anti-rabbit secondary antibody, MitoTracker, as well as DAPI. FITC, MitoTracker and DAPI fluorescent images were captured and are shown here. The scale bar indicates $10 \mu \mathrm{m}$. (TIF $15003 \mathrm{~kb}$ )

\section{Abbreviations \\ ALAS1: 5'-Aminolevulinate Synthase 1; BCC: basal cell carcinoma; \\ CycT: cyclopamine tartrate; DAPI: 4', 6-Diamidino-2-phenylindole; DCFH- DA: 2,7-dichlorodihydrofluorescein diacetate; GLUL: glutamate-ammonia ligase; HO1: heme oxygenase 1; MRPL23: mitochondrial ribosomal protein L23; NDUFS8: NADH Dehydrogenase (Ubiquinone) Fe-S Protein 8; NSCLC: non-small-cell lung cancer; PRDX6: peroxiredoxin 6; ROS: reactive oxygen species.}

\section{Competing interests}

Massoud Garrossian is the president of Logan Natural Products. No potential conflicts of interests were disclosed by the other authors.

\section{Authors' contributions}

Conception and design: MMA and LZ. Acquisition of data (measuring oxygen consumption, measuring cell growth and cell death, measuring ROS and membrane potential, Western blotting, fluorescence imaging, generating (ycT): MMA, SS, SPK and MG. Analysis and interpretation of data (e.g., statistical analysis, biostatistics, computational analysis): MMA and LZ. Writing, review, and/or revision of the manuscript: MMA and LZ. Administrative, technical, or material support (i.e., reporting or organizing data, constructing databases): MMA and LZ. Study supervision: LZ. All authors read and approved final manuscript. 


\section{Acknowledgments}

We thank Ms. Keely E Fitzgerald for her grammatical reviewing of this manuscript.

\section{Grant support}

This work was supported by the Cecil H. and Ida Green funds (LZ).

\section{Author details}

'Department of Molecular and Cell Biology, Center for Systems Biology, University of Texas at Dallas, Mail Stop RL11, 800 W. Campbell Road, Richardson, TX 75080, USA. ${ }^{2}$ Logan Natural Products, 2528 Royal Troon Dr, Plano, TX 75025, USA. ${ }^{3}$ The Cecil H. and Ida Green Distinguished Chair, Department of Biological Sciences, The University of Texas at Dallas, Mail Stop RL11, 800 W. Campbell Road, Richardson, TX 75080, USA.

\section{Received: 4 October 2015 Accepted: 17 February 2016} Published online: 24 February 2016

\section{References}

1. Briscoe J, Therond PP. The mechanisms of Hedgehog signalling and its roles in development and disease. Nat Rev Mol Cell Biol. 2013;14(7):416-29.

2. Hui CC, Angers S. Gli proteins in development and disease. Annu Rev Cell Dev Biol. 2011:27:513-37.

3. Karhadkar SS, Bova GS, Abdallah N, Dhara S, Gardner D, Maitra A, et al. Hedgehog signalling in prostate regeneration, neoplasia and metastasis. Nature. 2004;431(7009):707-12.

4. Berman DM, Karhadkar SS, Maitra A, Montes De Oca R, Gerstenblith MR,

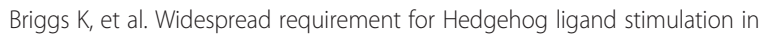
growth of digestive tract tumours. Nature. 2003;425(6960):846-51.

5. Watkins DN, Berman DM, Burkholder SG, Wang B, Beachy PA, Baylin SB. Hedgehog signalling within airway epithelial progenitors and in small-cell lung cancer. Nature. 2003;422(6929):313-7.

6. Jones S, Zhang X, Parsons DW, Lin JC, Leary RJ, Angenendt P, Mankoo P, Carter $\mathrm{H}$, Kamiyama $\mathrm{H}$, Jimeno $\mathrm{A}$, et al. Core signaling pathways in human pancreatic cancers revealed by global genomic analyses. Science. 2008;321(5897):1801-6.

7. Bhattacharya R, Kwon J, Ali B, Wang E, Patra S, Shridhar V, Mukherjee P. Role of hedgehog signaling in ovarian cancer. Clin Cancer Res. 2008;14(23):7659-66.

8. Stecca B, Mas C, Clement V, Zbinden M, Correa R, Piguet V, et al. Melanomas require HEDGEHOG-GLI signaling regulated by interactions between GLI1 and the RAS-MEKNAKT pathways. Proc Natl Acad Sci U S A. 2007;104(14):5895-900

9. Amakye D, Jagani Z, Dorsch M. Unraveling the therapeutic potential of the Hedgehog pathway in cancer. Nat Med. 2013;19(11):1410-22.

10. Di Magno L, Coni S, Di Marcotullio L, Canettieri G. Digging a hole under Hedgehog: downstream inhibition as an emerging anticancer strategy. Biochim Biophys Acta. 2015;1856(1):62-72.

11. Chen JK, Taipale J, Cooper MK, Beachy PA. Inhibition of Hedgehog signaling by direct binding of cyclopamine to Smoothened. Genes Dev. 2002;16(21): 2743-8.

12. Taipale J, Chen JK, Cooper MK, Wang B, Mann RK, Milenkovic L, Scott MP, Beachy PA. Effects of oncogenic mutations in Smoothened and Patched can be reversed by cyclopamine. Nature. 2000;406(6799):1005-9.

13. Thayer SP, di Magliano MP, Heiser PW, Nielsen CM, Roberts DJ, Lauwers GY, et al. Hedgehog is an early and late mediator of pancreatic cancer tumorigenesis. Nature. 2003:425(6960):851-6.

14. Berman DM, Karhadkar SS, Hallahan AR, Pritchard II, Eberhart CG, Watkins DN, et al. Medulloblastoma growth inhibition by hedgehog pathway blockade. Science. 2002;297(5586):1559-61.

15. Sanchez P, Ruiz i Altaba A. In vivo inhibition of endogenous brain tumors through systemic interference of Hedgehog signaling in mice. Mech Dev. 2005;122(2):223-30.

16. Winkler JD, Isaacs A, Holderbaum L, Tatard V, Dahmane N. Design and synthesis of inhibitors of Hedgehog signaling based on the alkaloid cyclopamine. Org Lett. 2009;11(13):2824-7.

17. Fan Q, Gu D, He M, Liu H, Sheng T, Xie G, Li CX, Zhang X, Wainwright B, Garrossian A et al. Tumor shrinkage by cyclopamine tartrate through inhibiting hedgehog signaling. Chin J Cancer. 2011:30(7):472-81.

18. Hooda J, Cadinu D, Alam MM, Shah A, Cao TM, Sullivan LA, Brekken R, Zhang $\mathrm{L}$. Enhanced heme function and mitochondrial respiration promote the progression of lung cancer cells. PLoS One. 2013;8(5):e63402.

19. Anastasiou D, Cantley LC. Breathless cancer cells get fat on glutamine. Cell Res. 2012;22(3):443-6.
20. Ward PS, Thompson CB. Metabolic reprogramming: a cancer hallmark even warburg did not anticipate. Cancer Cell. 2012;21(3):297-308.

21. Stanton BZ, Peng LF. Small-molecule modulators of the Sonic Hedgehog signaling pathway. Mol Biosyst. 2009:6(1):44-54.

22. Leem YE, Ha HL, Bae JH, Baek KH, Kang JS. CDO, an Hh-coreceptor, mediates lung cancer cell proliferation and tumorigenicity through Hedgehog signaling. PLoS One. 2014;9(11), e111701.

23. Zhang L. HEME BIOLOGY: The Secret Life of Heme in Regulating Diverse Biological Processes. Singapore: World Scientific Publishing Company; 2011.

24. Liu H, Jian Q, Xue K, Ma C, Xie F, Wang R, Liao W, Liu Y, Chi S, Li C. The MEK/ERK signalling cascade is required for sonic hedgehog signalling pathway-mediated enhancement of proliferation and inhibition of apoptosis in normal keratinocytes. Exp Dermatol. 2014:23(12):896-901.

25. McCarron JG, Wilson C, Sandison ME, Olson ML, Girkin JM, Saunter C, Chalmers S. From structure to function: mitochondrial morphology, motion and shaping in vascular smooth muscle. J Vasc Res. 2013;50(5):357-71.

26. Picard M, Shirihai OS, Gentil BJ, Burelle Y. Mitochondrial morphology transitions and functions: implications for retrograde signaling? Am J Physiol Regul Integr Comp Physiol. 2013;304(6):R393-406.

27. van der Bliek AM, Shen Q, Kawajiri S. Mechanisms of mitochondrial fission and fusion. Cold Spring Harb Perspect Biol 2013;5(6). doi: 10.1101/cshperspect.a011072

28. $\mathrm{Ng} \mathrm{JM}$, Curran T. The Hedgehog's tale: developing strategies for targeting cancer. Nat Rev Cancer. 2011;11(7):493-501.

29. Dlugosz A, Agrawal S, Kirkpatrick P. Vismodegib. Nat Rev Drug Discov. 2012; 11(6):437-8

30. Kovacevic Z. The pathway of glutamine and glutamate oxidation in isolated mitochondria from mammalian cells. Biochem J. 1971;125(3):757-63.

31. Moreadith RW, Lehninger AL. The pathways of glutamate and glutamine oxidation by tumor cell mitochondria. Role of mitochondrial NAD(P) + -dependent malic enzyme. J Biol Chem. 1984;259(10):6215-21.

32. Hensley CT, Wasti AT, DeBerardinis RJ. Glutamine and cancer: cell biology, physiology, and clinical opportunities. J Clin Invest. 2013;123(9):3678-84.

33. Moses MA, Neckers L. The GLU that holds cancer together: targeting GLUtamine transporters in breast cancer. Cancer Cell. 2015;27(3):317-9.

34. Kadenbach B, Ramzan R, Wen L, Vogt S. New extension of the Mitchell Theory for oxidative phosphorylation in mitochondria of living organisms. Biochim Biophys Acta. 2010;1800(3):205-12.

35. Korenic A, Boltze J, Deten A, Peters M, Andjus P, Radenovic L. Astrocytic mitochondrial membrane hyperpolarization following extended oxygen and glucose deprivation. PLoS One. 2014;9(2):e90697.

36. Kadenbach B, Ramzan $\mathrm{R}$, Moosdorf $\mathrm{R}$, Vogt $\mathrm{S}$. The role of mitochondrial membrane potential in ischemic heart failure. Mitochondrion. 2010;11(5):700-6.

37. Liu SS. Cooperation of a "reactive oxygen cycle" with the Q cycle and the proton cycle in the respiratory chain-superoxide generating and cycling mechanisms in mitochondria. J Bioenerg Biomembr. 1999;31(4):367-76.

38. Le Bras M, Clement MV, Pervaiz S, Brenner C. Reactive oxygen species and the mitochondrial signaling pathway of cell death. Histol Histopathol. 2005; 20(1):205-19.

39. Martinez-Reyes I, Cuezva JM. The H(+)-ATP synthase: a gate to ROS-mediated cell death or cell survival. Biochim Biophys Acta. 2014;1837(7):1099-112.

40. Otera H, Mihara K. Mitochondrial dynamics: functional link with apoptosis. Int J Cell Biol. 2012;2012:821676.

41. Lee EY, Ji H, Ouyang Z, Zhou B, Ma W, Vokes SA, et al. Hedgehog pathwayregulated gene networks in cerebellum development and tumorigenesis. Proc Natl Acad Sci U S A. 2010;107(21):9736-41.

42. Vokes SA, Ji H, McCuine S, Tenzen T, Giles S, Zhong S, et al. Genomic characterization of Gli-activator targets in sonic hedgehog-mediated neural patterning. Development. 2007;134(10):1977-89.

43. Vokes SA, Ji H, Wong WH, McMahon AP. A genome-scale analysis of the cis-regulatory circuitry underlying sonic hedgehog-mediated patterning of the mammalian limb. Genes Dev. 2008:22(19):2651-63.

44. Ramirez RD, Sheridan S, Girard L, Sato M, Kim Y, Pollack J, Peyton M, Zou Y, Kurie JM, Dimaio JM et al. Immortalization of human bronchial epithelial cells in the absence of viral oncoproteins. Cancer Res. 2004;64(24):9027-34

45. Whitehurst AW, Bodemann BO, Cardenas J, Ferguson D, Girard L, Peyton M, et al. Synthetic lethal screen identification of chemosensitizer loci in cancer cells. Nature. 2007;446(7137):815-9

46. Papandreou I, Cairns RA, Fontana L, Lim AL, Denko NC. HIF-1 mediates adaptation to hypoxia by actively downregulating mitochondrial oxygen consumption. Cell Metab. 2006:3(3):187-97. 